\title{
Functional correlates of self-reported energy levels in the Health, Aging and Body Composition Study
}

\author{
Rebecca Ehrenkranz ${ }^{1}$ (D ) Andrea L. Rosso ${ }^{1} \cdot$ Briana N. Sprague ${ }^{1} \cdot$ Qu Tian $^{2} \cdot$ Theresa Gmelin $^{1} \cdot$ Nicolaas Bohnen $^{3}$. \\ Eleanor M. Simonsick ${ }^{2} \cdot$ Nancy W. Glynn ${ }^{1} \cdot$ Caterina Rosano $^{1}$
}

Received: 25 September 2020 / Accepted: 6 January 2021 / Published online: 10 March 2021

(c) The Author(s) 2021

\begin{abstract}
Background Effects of fatigue on health in older age are well studied, yet little is known about the clinical relevance of energy perception.

Aims To explore cross-sectional associations of self-reported energy with physical and mental health metrics in the Health, Aging, and Body Composition Study.

Methods Participants rated their energy from 0 to 10; the outcome was energy dichotomized at the median ( $\geq 7=$ higher energy). Four domains were assessed: depressive symptoms (Center for Epidemiologic Studies Depression Scale); physical performance (function: usual and rapid gait speed; fitness: 400-m walk time); physical activity (casual walking, walking for exercise, and intense exercise); and cognitive function (Modified Mini-Mental State Examination and Digit Symbol Substitution Test). Covariates bivariately associated with energy entered a multivariable logistic regression model, adjusted for demographics, chronic conditions, and strength.

Results Depressive symptoms, physical performance and activity, but not cognition, were bivariately associated with energy $(p<0.0005)$. Younger age, male sex, greater strength, and absence of chronic conditions predicted higher energy $(p<0.001)$. In a multivariable model, depressive symptoms [adjusted odds ratio (aOR) 95\% CI $0.69(0.62,0.76)$ ] and 400-m walk times $[\mathrm{aOR}=0.81(0.72,0.91)]$ were inversely associated with energy; usual and rapid gait speed $[\mathrm{aOR}=1.3(1.2,1.4) ; \mathrm{aOR}=1.2$ (1.1-1.4)], and time spent in intense exercise $[\mathrm{aOR}=1.4(1.1-1.7)]$ were positively associated with energy.

Discussion In this cohort with a range of chronic conditions and fatigue, perceiving higher energy levels may reflect better emotional and physical health.
\end{abstract}

Conclusion Energy should be considered in multidimensional clinical assessments of older age.

Keywords Energy $\cdot$ Fatigue $\cdot$ Successful aging $\cdot$ Epidemiology

Supplementary Information The online version contains supplementary material available at https://doi.org/10.1007/ s40520-021-01788-0.

Rebecca Ehrenkranz

ree24@pitt.edu

1 Department of Epidemiology, Graduate School of Public Health, University of Pittsburgh, Pittsburgh, PA, USA

2 Intramural Research Program, National Institute on Aging, Baltimore, MD, USA

3 Department of Neurology, University of Michigan, Ann Arbor, MI, USA

\section{Introduction}

Consequences of aging are often studied and framed in relation to deleterious health outcomes, but less is known about the clinical relevance of maintaining higher levels of energy later in life. Recent research suggests that one's perception of his or her energy level may be an indicator of global health status [1-3]. Reporting higher levels of energy may also reflect general life satisfaction and continued social participation [3, 4]. To date, little research describes the physical and mental health characteristics associated with higher levels of energy in older adults. Notably, fatigue, defined as lack of energy, is commonly evaluated in aging studies and its negative association with health outcomes have been 
reported [5-8]. However, the absence of fatigue does not necessarily affirm the presence of higher energy levels.

Energy and fatigue are often viewed as opposites on a continuum, yet there is increasing evidence they have different neurobiological correlates [9-11]. For example, recent research suggests alterations in dopamine are associated with changes in energy but not fatigue [9]. Conversely, increased serotonin levels have been linked to fatigue but not to energy $[9,12]$. In a small double-blind placebo controlled trial in participants under age 40 , those given a histamine precursor reported reduced fatigue with no changes in energy [ 9 , 13]. These results indicate that energy and associated health characteristics should be evaluated separately from fatigue.

Energy can be measured objectively or subjectively. Objective measures primarily capture traits or physical fitness [14-16]; whereas, subjective measures capture energy state as well as perception of that state. Emerging evidence indicates subjective measures and individual perceptions are important predictors of clinically relevant outcomes [17-19]. For example, those who self-report favorable perceptions of aging had faster walking speed, better memory, and longer life span than those with unfavorable perceptions of aging [18, 20-22]. Analogously, self-reported energy may be associated with similar health-relevant characteristics.

In this study, we examined older adults' objective measures of physical and mental health in relation to self-reported energy levels in a cohort of community-dwelling men and women with a range of chronic conditions. We hypothesized that lower depressive symptoms, higher physical performance, higher levels of fitness and physical activity, and better cognitive function would be associated with higher self-reported energy.

\section{Methods}

The Health, Aging and Body Composition (Health ABC) Study is a longitudinal cohort study that enrolled 3075 Black and White community-dwelling adults aged 70-79 between March 1997 and July 1998 with the overarching objective to evaluate risk factors and disparities in the onset of functional limitation in healthy older adults. To be eligible, participants at baseline had to be free of reported difficulty walking $1 / 4$ mile or climbing 10 steps [23]. Health $\mathrm{ABC}$ provides the opportunity to examine both singular and composite associations between common, related factors (such as body mass index) and energy expenditure and fatigue.

In the current analyses, we selected those who answered the question about self-reported energy over the past month at Year Three (calendar years 1999-2000) of the study. All other variables are derived from Year Three, except for time to walk $400 \mathrm{~m}$ (assessed at Year Two) and the following variables ascertained at baseline: race, sex, education, peripheral artery disease, and Digit Symbol Substitution Test (DSST) results. Arthritis, cancer, diabetes, and cardiovascular conditions were assessed annually, and prevalence of these conditions was calculated at Year 3.

\section{Self-reported energy}

Self-reported energy, the main outcome, was captured as follows: "Using this card, please choose the category that best describes your usual energy level in the past month on a scale of 0 to 10 where 0 is no energy and 10 is the most energy that you have ever had."

\section{Main independent variables: physical and mental characteristics of interest}

Depressive symptomatology was assessed using the Center for Epidemiologic Studies Depression Scale Revised 10 (CESD-10) questionnaire [24]. CESD-10 scores $\geq 11$ indicate clinical depressive symptoms, scores between 5 and 10 indicate subclinical depressive symptoms, and scores $<5$ indicate normal mood [25]. A modified CESD-10 score was computed for sensitivity analysis after exclusion of two questions that could be interpreted as related to energy and fatigue: "I felt that everything I did was an effort" and "I cannot get going" [26].

Physical performance was captured with measures of physical function and physical fitness. Physical function was measured as usual and rapid-pace gait speed (meters/ second) during a 20-m walk at Year 3. Physical fitness was assessed as the time it took a participant to walk $400 \mathrm{~m}$ as quickly as possible, with longer walk times consistent with lower fitness [27]; a total of $n=1715$ (68\% of the sample) completed this test.

Physical activity was measured by asking participants about their walking habits in the prior week, and their answers were converted to total minutes of walking per week. Participants were also asked if over the last 12 months they did any of the following at least 10 times: walked for exercise, walked to complete day-to-day tasks, climbed a flight of stairs, and engaged in high-intensity exercise; answers were recorded as yes/no.

Cognitive function was assessed via the Modified MiniMental State (3MS) Examination and Digit Symbol Substitution Test. The 3MS is a screening test for cognitive impairment with a score range of $0-100$, with higher scores indicating better cognitive function [28]. 3MS score cutoffs for clinical disorders are scores $<80$ points, subclinical disorder cut-offs are $80-85$ points, and normal status scores are $>85[25,29]$. The DSST is a neuropsychiatric test of psychomotor performance which involves matching symbols to numbers [30]. DSST score is the total number of accurate symbol-number matches made in $90 \mathrm{~s}[25,30]$. 


\section{Fatigue and other health-related measures of interest}

Fatigue was captured by asking the question: "In the past month, on average, have you been feeling unusually tired during the day?" Response options were yes or no, and if yes, participants reported if they felt unusually tired: all of the time, most of the time, or some of the time. Responses were grouped as yes or no for the analysis.

Demographic variables included chronological age, sex, race and highest level of education (all collected via selfreport). Prevalent, pre-existing chronic conditions included: cardiovascular disease, diabetes, cancer, peripheral arterial disease, and osteoarthritis. These were measured via selfreport questionnaires, medications listed, or Health Care Finance Administration diagnosis. A composite "cardiovascular conditions" variable was created to capture the presence of any of the following: coronary disease, chronic heart failure, cardiovascular disease, or hypertension. Anthropometric measures included: body mass index (BMI, measured as $\left.\mathrm{kg} / \mathrm{m}^{2}\right]$ and quadriceps strength, measured as peak torque results from Kin-Com dynamometer [31].

\section{Statistical analyses}

In this cross-sectional analysis, energy was the dependent variable; depressive symptoms, physical performance and activity, and cognition were the main independent variables. Self-reported energy was skewed towards higher scores and dichotomized at the median into higher and lower energy (Supplemental Figure 1). Given that participants may have interpreted the energy question differently based on personality or other factors, dichotomizing self-reported energy served to minimize potential variation and may improve the likelihood that those in the higher energy group truly had greater self-reported energy than those in the lower energy group. $T$ tests or Chi-square tests were used to evaluate the associations of continuous or categorical variables with energy, respectively.

When significant $(p<0.05)$, bivariate associations for the main independent variables were found, we included these variables in multivariable logistic regression models with energy level as the outcome. Each main predictor entered one regression model at a time, with further adjustment for age and sex and further adjustment for covariates. Selection of covariates was based on bivariate associations with selfreported energy at $p<0.05$. All main independent measures associated with energy entered a final combined model. Continuous independent variables were in standardized units.

Sensitivity analyses assessed whether results differed when including the following variables in the models: (a) fatigue (b) a modified CES-D score, excluding the two energyrelated questions; (b) clinical cut-offs for CESD-10 (normal: scores $<5$, subclinical depressive symptoms: scores 5-10, and clinically depressive symptoms: scores $\geq 11$ ); (c) clinical cutoffs for 3MS (normal: scores $>85$, impaired: scores $\leq 85$ ); (d) clinical cut-offs of usual gait speed (normal: speed $\geq 1.0 \mathrm{~m} /$ sec, impaired: $0.6<$ speed $<1.0 \mathrm{~m} / \mathrm{sec}$, disabled: speed $\leq 0.6 \mathrm{~m} /$ $\mathrm{sec})$; (e) data from those who completed the $400 \mathrm{~m}$ walk; that is, all models were repeated in the subsample of $n=1715$.

\section{Results}

Participants were aged $76 \pm 2.8$ years, with an even distribution of men and women, a slightly higher proportion of Whites than Blacks, and less than half with post-secondary education (Table 1). Older participants, women, and those with less education were more likely to be missing the energy variable $(p<0.05)$.

Observed self-reported energy ranged from the lowest possible score of 0 to the highest possible score of 10 and $58 \%$ were categorized as having higher energy (Table 1). Prevalence of pre-existing chronic conditions such as composite cardiovascular disease $(61.7 \%)$ and cancer $(21.5 \%)$ was similar to what is observed in community-based samples of this age [32-34]. Similarly, average values of depressive symptoms, physical and cognitive function were as expected for community-dwelling adults in this age group [25, 35, 36 ]. Around $7 \%$ had clinically depressive symptoms, $20 \%$ had cognitive impairment based on 3MS score, and $24 \%$ had a gait speed $<1 \mathrm{~m} / \mathrm{sec}$. More than half of the sample reported walking for exercise $(65.4 \%, n=1655)$ and around a quarter of the sample reported undertaking high-intensity exercise $(26.6 \%, n=671)$.

\section{Results of bivariate associations}

Fewer depressive symptoms, higher physical function and activity were bivariately associated with higher energy at $p<0.001$ (Table 1). Associations were similar for the modified CESD-10 score that excludes the fatigue-related items. Younger age, being male, having higher muscle strength, and presence of any pre-existing chronic conditions except cancer were also bivariately associated with higher energy at $p<0.05$ (Table 1). Although higher energy and lower fatigue (unusual tiredness) were significantly negatively correlated (correlation coefficient $=-0.33, p<0.001$ ), a relatively high proportion of those reporting higher energy also reported fatigue (40\%).

\section{Results of separate multivariable logistic regression models predicting higher energy}

The association of CESD-10 score with higher energy was independent of all covariates. For each standard deviation increase in CESD-10 score, there was approximately a 25\% 
Table 1 Sample characteristics and associations with higher and lower self-reported energy in the Health, Aging and Body Composition Study $(N=2529)$

\begin{tabular}{|c|c|c|c|c|}
\hline Characteristic & $\begin{array}{l}\text { Total sample } \\
N=2529 \text { Mean } \pm \text { SD } \\
\text { or } \mathrm{N}(\%)\end{array}$ & $\begin{array}{l}\text { Higher energy } \\
N=1462 \text { Mean } \pm \text { SD } \\
\text { or N }(\%)\end{array}$ & $\begin{array}{l}\text { Lower energy } \\
N=1067 \text { Mean } \pm \mathrm{SD} \\
\text { or } \mathrm{N}(\%)\end{array}$ & $p$ value \\
\hline \multicolumn{5}{|l|}{ Demographics } \\
\hline Age in years & $75.6 \pm 2.8$ & $75.4 \pm 2.8$ & $75.9 \pm 2.9$ & 0.0002 \\
\hline Men & $1,223(48.4)$ & $744(50.9)$ & 479 (44.9) & 0.003 \\
\hline White & $1,555(61.5)$ & $878(60.1)$ & $677(63.5)$ & 0.08 \\
\hline Post-secondary education & $1,121(44.5)$ & $655(44.9)$ & $466(43.8)$ & 0.6 \\
\hline \multicolumn{5}{|l|}{ Depressive symptoms and fatigue } \\
\hline CESD-10 $0^{\mathrm{a}}$ score & $7.6 \pm 3.1$ & $7.0 \pm 2.6$ & $8.3 \pm 3.5$ & $<0.0001$ \\
\hline Modified CESD-10 score ${ }^{*}$ & $6.8 \pm 2.5$ & $6.5 \pm 2.2$ & $7.1 \pm 2.8$ & $<0.0001$ \\
\hline Any unusual tiredness & $621(24.6)$ & 199 (13.6) & $422(40.0)$ & $<0.0001$ \\
\hline \multicolumn{5}{|l|}{ Physical function } \\
\hline Usual gait speed, m/sec & $1.15 \pm 0.22$ & $1.18 \pm 0.21$ & $1.10 \pm 0.22$ & $<0.0001$ \\
\hline Rapid gait speed, m/sec & $1.52 \pm 0.34$ & $1.56 \pm 0.33$ & $1.46 \pm 0.34$ & $<0.0001$ \\
\hline \multicolumn{5}{|l|}{ Physical fitness } \\
\hline Time to walk $400 \mathrm{~m}, \mathrm{~m} / \mathrm{sec}^{\mathrm{b}}$ & $328.6 \pm 60.4$ & $321 \pm 59.4$ & $339.6 \pm 60.3$ & $<0.0001$ \\
\hline \multicolumn{5}{|l|}{ Physical activity } \\
\hline Walking for exercise (past 12 months, $\geq 10$ times) & $1,655(65.4)$ & $998(68.5)$ & $654(61.4)$ & $<0.0001$ \\
\hline High-intensity exercise (past 12 months, $\geq 10$ times) & $671(26.6)$ & $426(29.2)$ & $244(22.9)$ & $<0.0001$ \\
\hline Time spent walking weekly, minutes & $102.8 \pm 175$ & $113.6 \pm 183$ & $88.1 \pm 163$ & 0.0003 \\
\hline \multicolumn{5}{|l|}{ Cognitive function } \\
\hline Teng $3 \mathrm{MS}^{\mathrm{c}}$ & $89.9 \pm 8.8$ & $90.0 \pm 8.5$ & $90.0 \pm 8.9$ & 0.9 \\
\hline Digit symbol substitution score & $36.6 \pm 14.3$ & $36.61 \pm 14.9$ & $36.61 \pm 13.6$ & 0.9 \\
\hline \multicolumn{5}{|l|}{ Pre-existing health conditions } \\
\hline Diabetes, prevalent & $443(17.5)$ & $232(15.9)$ & $211(19.8)$ & 0.01 \\
\hline Cancer, ever & $541(21.5)$ & $291(20.0)$ & $250(23.5)$ & 0.034 \\
\hline Cardiovascular conditions, prevalent ${ }^{\mathrm{d}}$ & $1560(61.7)$ & $856(58.6)$ & $704(66.0)$ & $<0.0001$ \\
\hline Peripheral arterial disease, prevalent & $120(4.9)$ & $49(3.4)$ & $71(6.9)$ & $<0.0001$ \\
\hline Arthritis, prevalent & $969(38.3)$ & $498(34.1)$ & $471(44.1)$ & $<0.0001$ \\
\hline \multicolumn{5}{|l|}{ Anthropometric measures } \\
\hline Peak torque $($ mean $\pm \mathrm{SD})$ & $78.0 \pm 29.1$ & $80.3 \pm 28.9$ & $74.3 \pm 28.9$ & $<0.0001$ \\
\hline Body mass index, $\mathrm{kg} / \mathrm{m} 2($ mean $\pm \mathrm{SD})$ & $27.2 \pm 4.8$ & $27.1 \pm 4.7$ & $27.3 \pm 4.8$ & 0.4 \\
\hline
\end{tabular}

Distributions of participant characteristics by energy group. $T$ tests were used for continuous variables, and Chi-square tests for categorical variables

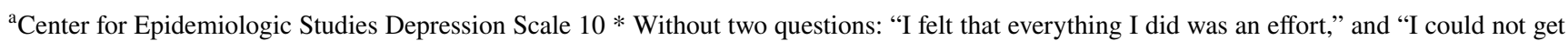
going"

${ }^{\mathrm{b}}$ Time to walk $400 \mathrm{~m}$ was only recorded for 1,715 participants who completed the walk successfully

${ }^{\mathrm{c}}$ Teng Mini-Mental State Exam

${ }^{\mathrm{d}}$ Cardiovascular disease was a composite variable including: coronary heart disease, chronic heart failure, cardiovascular disease, and hypertension

lower odds of reporting higher energy in fully adjusted models $[\mathrm{aOR}=0.69(0.62-0.76)]$. Results were similar across all model iterations (Table 2); results were attenuated when using the modified CES-D $[\mathrm{aOR}=0.84(0.76-0.92)]$ that excludes the fatigue-related items.

All measures of physical function (performance and fitness) were associated with higher energy. Faster usual and rapid gait speed over $20 \mathrm{~m}$ were associated with greater odds of higher self-reported energy [aOR $=1.3(1.2-1.5)$ and $\mathrm{aOR}=1.2(1.1-1.4)$ in fully adjusted models, respectively, or 30-20\% difference for each standard deviation of the independent variable]. Longer time to walk $400 \mathrm{~m}$ was associated with lower odds of reporting higher energy in fully adjusted models $[\mathrm{aOR}=0.81(0.72-0.91)$ ], or a $20 \%$ difference for each standard deviation of the independent variable. Results were significant in all model iterations (Table 2). 
Table 2 Odds ratios and 95\% confidence intervals of separate multivariable logistic regression models of mood and physical function associated with higher energy in the Health, Aging and Body Composition Study $(N=2529)$

\begin{tabular}{|c|c|c|c|c|}
\hline Independent variable ${ }^{\mathrm{a}}$ & Unadjusted & Adjusted for age and sex & $\begin{array}{l}\text { Further adjusted for pre-existing } \\
\text { chronic conditions }{ }^{b}\end{array}$ & $\begin{array}{l}\text { Further adjusted } \\
\text { for muscle } \\
\text { strength }\end{array}$ \\
\hline Depressive symptoms & $0.65(0.60-0.71)$ & $0.66(0.61-0.72)$ & $0.68(0.62-0.75)$ & $0.69(0.62-0.76)$ \\
\hline Usual walk speed & $1.5(1.3-1.6)$ & $1.4(1.3-1.5)$ & $1.4(1.2-1.5)$ & $1.3(1.2-1.4)$ \\
\hline Rapid walk speed & $1.4(1.3-1.5)$ & $1.4(1.3-1.5)$ & $1.3(1.2-1.4)$ & $1.2(1.1-1.3)$ \\
\hline Time to walk $400 \mathrm{~m}^{\mathrm{c}}$ & $0.72(0.65-0.80)$ & $0.74(0.66-0.82)$ & $0.76(0.68-0.84)$ & $0.80(0.71-0.91)$ \\
\hline Minutes walking per week & $1.2(1.1-1.3)$ & $1.1(1.0-1.2)$ & $1.1(1.0-1.2)$ & $1.1(0.99-1.2)$ \\
\hline Walking for exercise & $1.4(1.2-1.6)$ & $1.3(1.1-1.6)$ & $1.3(1.1-1.5)$ & $1.3(1.0-1.5)$ \\
\hline High intensity exercise & $1.4(1.2-1.7)$ & $1.4(1.1-1.6)$ & $1.4(1.1-1.6)$ & $1.4(1.1-1.7)$ \\
\hline
\end{tabular}

Odds ratios for each independent variable in progressive model iterations. Depressive symptoms, usual and rapid walk speed, time to walk $400 \mathrm{~m}$, and high-intensity exercise were significantly associated with higher energy in each iteration

${ }^{a}$ Each row reports results of separate models, where the variable listed in the first column is the main independent variable, and the model is progressively adjusted for covariates that were bivariately associated with energy. Note: the main independent variables are in standardized units

${ }^{\mathrm{b}}$ Adjusted for: peripheral artery disease, diabetes, cardiovascular disease, cancer, arthritis

${ }^{\mathrm{c}}$ Time to walk $400 \mathrm{~m}$ was recorded for 1715 participants who completed the walk successfully

More physical activity was associated with higher energy, with stronger associations for walking for exercise and engaging in high-intensity exercise (30-40\% difference for each standard deviation of the independent variable) compared to walking to complete day-to-day tasks (about a 10\% difference for each standard deviation of the independent variable).

Neither measure of cognitive function was associated with energy in either unadjusted or adjusted models. The odds ratios for both remained at or below 1.0 as covariates were progressively added to each model (not shown). None of the interactions tested were significant $(p>0.1$ for all).

\section{Results of the final multivariable logistic regression model predicting higher energy and sensitivity analyses}

A final model to evaluate higher self-reported energy included all independent variables that were associated with energy in separate multivariable models described above. Of note, this model was limited to the subgroup of those who completed the 400-m walk (subsample of $n=1715$ ). In this model, only CESD-10, 400-m walk time, and time spent engaged in high-intensity exercise remained associated with energy (Fig. 1). In this model, the coefficients of age, cardiovascular disease, arthritis, and muscle strength were also statistically significant (Supplemental Table 1). Results were similar when using the modified CES-D (not shown).

Sensitivity analyses controlling for fatigue yielded similar results for each model (not shown). Using clinical cut-offs for CESD-10 (with all questions included) and no depressive symptoms as the reference category yielded results where subclinical depressive scores were not significantly associated with energy [aOR $=0.95(0.75-1.2)]$, but clinically depressive scores were strongly associated with energy [aOR $=0.42(0.30-0.57)]$. Clinical cut-off categories for $3 \mathrm{MS}$ did not yield significant results in either bivariate or multivariable models.

\section{Discussion}

Our results suggest that self-reported energy may reflect a person's emotional and physical health (depressive symptoms, gait speed and fitness), as well as the extent to which a person would make use of his or her capacity in everyday life (walking and other exercise). These associations were robust to adjustment for other energy-related factors, including demographic characteristics, pre-existing chronic conditions, muscle strength, and fatigue. This analysis addresses a gap in our understanding of fatigue, energy and health characteristics in older age, by examining associations between objective cognitive and physical performance metrics and energy perception. Our data indicate that energy and fatigue are not entirely overlapping domains. In this sample, among those who reported higher energy, $40 \%$ also reported unusual tiredness over the last month, indicating that one is not simply the absence of the other. This distinction may be because energy and fatigue states are driven by different underlying neurobiological mechanisms [9].

Our results that depressive symptoms are inversely related with energy is consistent with prior work in clinical and patients' populations. Reporting feelings of low energy are common symptoms of depressive disorders, especially in older age [36-39]. For example, one study found that decreased activity levels and increased apathy (defined as 
Fig. 1 Correlates of higher selfreported energy in the Health, Aging and Body Composition Study $(N=1715)$. Figure legend: In a model including all significant correlates of higher energy (depressive symptoms, usual and rapid gait, 400-m walk times, and high-intensity exercise), depressive symptoms, 400-m walk time, and intense exercise remained associated with higher self-reported energy

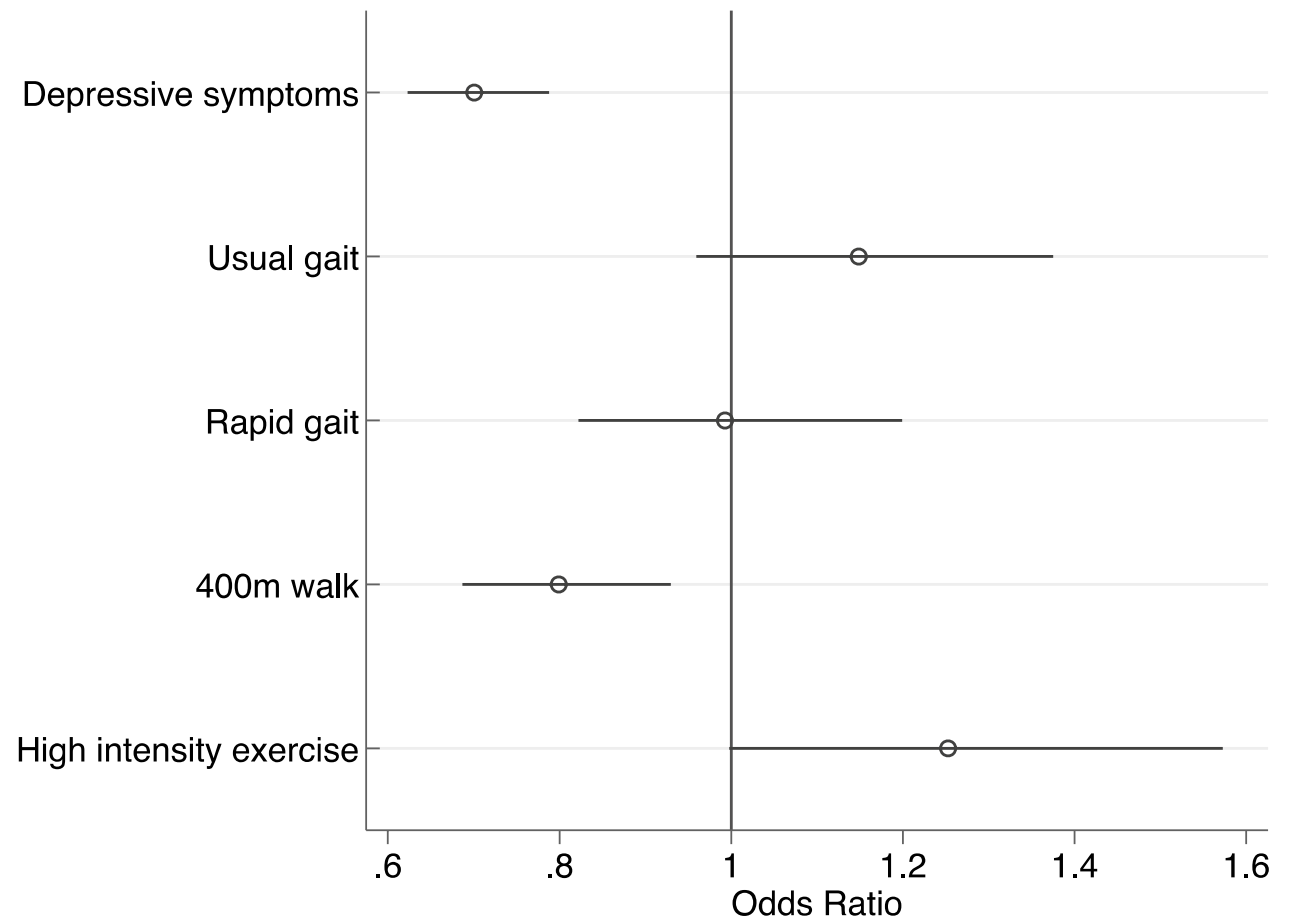

lack of motivation and affective dullness) was present in $75 \%$ of older depressed individuals [40]. Sensitivity analyses with clinical cut-off indicated associations are driven by more severe depressive symptoms. Of note, results were only partially attenuated when using the modified CESD10 , which excluded fatigue-related items. Although depressive symptoms are correlated with increased risk of reduced functional ability [41-43], accurate early detection of depression remains challenging, especially for older adults who do not openly disclose their emotional status to their physician. Future studies should examine whether reporting lower energy may be an accurate indicator of underlying depressive symptoms.

Our analyses advance our understanding of the link between physical function and energy. Much of the prior research on energy and gait has focused on objective measures of energy expenditure [40, 44, 45], but the results of this analysis indicate that gait speed has also robust associations with self-reported higher energy in the context of healthy aging. The $400 \mathrm{~m}$ walk can discriminate walking ability and fitness and can be used to identify early signs of functional decline in older adults [46]. Taken together, our results suggest that physical capacity is a distinct phenotypic characteristic of older adults reporting higher energy levels.

Across metrics of physical activity, those who reported exercising were more likely to also report higher selfreported energy than those who did not exercise. This relationship was not significant for those who reported engaging in day-to-day physical activity (such as walking to complete basic tasks or climbing stairs). It has been demonstrated that exercise can increase energy levels, and exercise has multiple mechanisms of action in the central nervous system that may directly affect energy perception [9, 47, 48]. Physical activity levels may be predictive of improved perception of higher energy level and may signal quality of life status in aging populations [44, 49]. These dose-response relationships parallel the results presented here, wherein higher levels of physical activity had the strongest associations with higher self-reported energy.

Contrary to our hypothesis, neither the $3 \mathrm{MS}$ nor the DSST was significantly associated with self-reported energy. There is little available literature on the connection of either of these two tests to energy. Reports of cognitive function tests and fatigue are often focused on vulnerable population subgroups (such as cancer patients or Parkinson's patients) and thus, results may not be applicable to healthy older adults. The $3 \mathrm{MS}$ may not be the most appropriate test to capture the range of cognitive abilities in well-functioning populations due to the ceiling effect $[50,51]$. It is possible that self-reported energy is related to other cognitive domains that are only partially captured by these tests, such as attention, inhibition, or task-switching.

\section{Limitations and strengths}

Conceptualizations of energy may differ between participants, leading to variability between individual energy score rankings; we tried to temperate this limitation by dichotomizing the values at the median value. The trade-off is that by dichotomizing we may have lost 
statistical power to detect a difference where one exists. Self-reported fatigue was asked right before self-reported energy, which may have influenced participants to report lower than actual energy. This potential misclassification may have biased results towards the null if it made those reporting lower energy more similar on characteristics of interest to those reporting higher energy. Objective metrics of energy expenditure were not available. This was a crosssectional evaluation of self-reported energy, so temporality and evaluating longitudinal changes in energy were beyond the scope of this analysis. As this was a healthy and relatively active cohort, results may be more generalizable to healthy older adults rather than the entire population of older adults. Of note, within this sample, those who were able to complete the $400 \mathrm{~m}$ walk may have had better overall health than those who were not. Other limitations pertain to the assessment of physical activity. Participants were asked about their levels of activity over the past year and may not accurately remember variations in day-to-day activity; participants may also have been more likely to recollect intentional exercise than time spent walking for general purposes. If so, results for high-intensity exercise may be biased away from the null. Additionally, participants were asked about their physical activity over multiple fairly similar questions, and their answers and accuracy may have varied depending on which questions were more meaningful to the individual participant.

A strength of this study is that it included numerous functional, performance-based, clinical and laboratory measures, including information on fatigue, as well as disease states. While there is substantial literature elucidating relationships between fatigue and aging, this analysis fills a gap by examining associations between objective cognitive and physical performance metrics and energy perception. For example, in this sample, among those who reported higher energy, $40 \%$ also reported unusual tiredness over the last month, indicating that one is not simply the absence of the other. These results build upon recent findings that there is a relationship between self-reported energy and physical activity by both objective and subjective measures [44]. Additionally, results from the Health $\mathrm{ABC}$ cohort are generalizable to well-functioning older adults living independently in the community. Numerous clinical measures were available, including information on both disease states and body composition. While it was not unexpected that pre-existing chronic conditions were associated with lower energy, we also noted a lack of significant association for the anthropometric measures BMI and muscle strength. This may indicate that body composition does not reflect energy in the way that physical function and activity do.

\section{Conclusion}

Our results indicate that when reporting on their recent energy levels, older adults may be referring to physical performance and emotional wellbeing, independent of pre-existing chronic conditions. This has direct relevance to the psychosocial framework of aging, as self-reported energy may be indicative of underlying mood and physical characteristics that can either reduce or improve quality of life. By including self-reported energy as part of multidimensional clinical assessments in older adult populations, there may be opportunities to develop new interventions around modifiable behaviors that can lead to increased energy and lower risk for diminished functional capacity. Directions for future research may include evaluating selfreported energy as a predictor of functional impairment or mortality, and should include longitudinal measures of energy perception in older adults.

Author contributions E, R, S, G, R: study planning and design, results interpretation, preparation of manuscript. S, T, G, B: analysis and results interpretation, preparation of manuscript. All authors discussed the results and contributed to writing and editing the manuscript per ICJME criteria for authorship.

Funding This research was supported by National Institute on Aging (NIA) Contracts N01-AG-6-2101; N01-AG-6-2103; N01-AG-6-2106; NIA grant R01-AG028050, and NINR grant R01-NR012459. This research was funded in part by the Intramural Research Program of the NIH, National Institute on Aging.

Data availability Data are publicly available; see the Health $\mathrm{ABC}$ website, Analysis Proposals section: https://healthabc.nia.nih.gov/analysisproposals-publications.

\section{Compliance with ethical standards}

Conflict of interest No authors have conflicts of interest to report.

Ethical approval All procedures performed in the study were in accordance with the ethical standards of the 1964 Helsinki declaration and its later amendments.

Informed consent Informed consent was obtained from all individual participants included in the study.

Open Access This article is licensed under a Creative Commons Attribution 4.0 International License, which permits use, sharing, adaptation, distribution and reproduction in any medium or format, as long as you give appropriate credit to the original author(s) and the source, provide a link to the Creative Commons licence, and indicate if changes were made. The images or other third party material in this article are included in the article's Creative Commons licence, unless indicated otherwise in a credit line to the material. If material is not included in the article's Creative Commons licence and your intended use is not permitted by statutory regulation or exceeds the permitted use, you will need to obtain permission directly from the copyright holder. To view a copy of this licence, visit http://creativecommons.org/licenses/by/4.0/. 


\section{References}

1. Seeman TE et al (1994) Predicting changes in physical performance in a high-functioning elderly cohort: macarthur studies of successful aging. J Gerontol 49:M97-M108

2. Rowe JW, Kahn RL (1987) Human aging: usual and successful. Science 237:143-149

3. Bowling A, Dieppe $\mathrm{P}$ (2005) What is successful ageing and who should define it? Br Med J 331:1548-1551

4. Conflating time and energy: views from older adults in lower socioeconomic status areas on physical activity in: journal of aging and physical activity volume 26 issue 3 (2018). Available at: https://journals-humankinetics-com.pitt.idm.oclc.org/ view/journals/japa/26/3/article-p506.xml?tab_body=pdf-6614. (Accessed: 4th May 2020)

5. Knoop V, Costenoble A, Vella Azzopardi R, Vermeiren S, Debain A, Jansen B, Scafoglieri A, Bautmans I, Gerontopole Brussels Study Group (2019) The operationalization of fatigue in frailty scales: a systematic review. Ageing Res Rev 53:100911. https://doi. org/10.1016/j.arr.2019.100911

6. Zengarini E et al (2015) Fatigue: relevance and implications in the aging population. Exp Gerontol 70:78-83

7. Avlund K (2010) Fatigue in older adults: an early indicator of the aging process? Aging Clin Exp Res 22:100-115

8. Tralongo P, Respini D, Ferraù F (2003) Fatigue and aging. Crit Rev Oncol Hematol 48(Suppl):S57-S64

9. Loy BD, Cameron MH, O'Connor PJ (2018) Perceived fatigue and energy are independent unipolar states: supporting evidence. Med Hypotheses 113:46-51

10. Deng N, Guyer R, Ware JE (2015) Energy, fatigue, or both? A bifactor modeling approach to the conceptualization and measurement of vitality. Qual Life Res 24:81-93

11. Dishman RK, Thom NJ, Puetz TW et al (2010) Effects of cycling exercise on vigor, fatigue, and electroencephalographic activity among young adults who report persistent fatigue. Psychophysiology 47:1066-1074

12. Kikuchi $\mathrm{H}$ et al (2010) Decreased blood serotonin in the premenstrual phase enhances negative mood in healthy women. J Psychosom Obstet Gynecol 31:83-89

13. Loy BD, O'Connor PJ (2016) The effect of histamine on changes in mental energy and fatigue after a single bout of exercise. Physiol Behav 153:7-18

14. Westerterp KR (2018) Changes in physical activity over the lifespan: impact on body composition and sarcopenic obesity. Obes Rev 19:8-13

15. Speakman JR, Westerterp KR (2010) Associations between energy demands, physical activity, and body composition in adult humans between 18 and 96 y of age. Am J Clin Nutr 92:826-834

16. Pes GM, Dore MP, Errigo A et al (2018) Analysis of physical activity among free-living nonagenarians from a sardinian longevous population. J Aging Phys Act 26:254-258

17. Westerhof GJ et al (2014) The influence of subjective aging on health and longevity: a meta-analysis of longitudinal data. Psychol Aging 29:793-802

18. Wurm S, Diehl M, Kornadt AE et al (2017) How do views on aging affect health outcomes in adulthood and late life? Explanations for an established connection. Dev Rev 46:27-43

19. Schroyen S, Letenneur L, Missotten P et al (2020) Impact of selfperception of aging on mortality of older patients in oncology. Cancer Med. https://doi.org/10.1002/cam4.2819

20. Kotter-Grühn D, Kleinspehn-Ammerlahn A, Gerstorf D et al (2009) Self-perceptions of aging predict mortality and change with approaching death: 16-year longitudinal results from the berlin aging study. Psychol Aging 24:654-667

21. Rippon I, Steptoe A (2015) Feeling old vs. being old: associations between self-perceived age and mortality. JAMA Intern Med 175:307-309
22. Sargent-Cox KA, Anstey KJ, Luszcz MA (2012) The relationship between change in self-perceptions of aging and physical functioning in older adults. Psychol Aging 27:750-760

23. Georgiopoulou VV, Kalogeropoulos AP, Chowdhury R et al (2017) Exercise capacity, heart failure risk, and mortality in older adults: the health ABC study. Am J Prev Med 52:144-153

24. Radloff LS (1977) The CES-D scale. Appl Psychol Meas 1:385-401

25. Rosano C et al (2016) Digit symbol substitution test and future clinical and subclinical disorders of cognition, mobility and mood in older adults. Age Ageing 45:688-695

26. Center for epidemiologic studies depression scale revised (CESD-R-10)

27. Newman $\mathrm{AB}$ et al (2006) Association of long-distance corridor walk performance with mortality, cardiovascular disease, mobility limitation, and disability. J Am Med Assoc 295:2018-2026

28. Teng EL, Chui HC (1987) The modified mini-mental state (MMS) examination. J Clin Psychiatry 48:314-318

29. Espeland MA et al (2006) Benchmarks for designing two-stage studies using modified mini-mental state examinations: experience from the women's health initiative memory study. Clin Trials 3:99-106

30. Jaeger J (2018) Digit symbol substitution test. J Clin Psychopharmacol 38:513-519

31. Mayhew TP, Rothstein JM, Finucane SD et al (1994) Performance characteristics of the Kin-Com ${ }^{\circledR}$ dynamometer I physical therapy I Oxford academic. Phys Ther 74:1047-1054

32. Noale M, Limongi F, Maggi S (2020) Epidemiology of cardiovascular diseases in the elderly. Adv Exp Med Biol 1216:29-38

33. White $\mathrm{MC}$ et al (2014) Age and cancer risk: a potentially modifiable relationship. Am J Prev Med 46:S7

34. Atella V, Piano Mortari A, Kopinska J, Belotti F, Lapi F, Cricelli C, Fontana L (2019) Trends in age-related disease burden and healthcare utilization. Aging Cell 18:e12861

35. Balsamo M, Cataldi F, Carlucci L et al (2018) Assessment of latelife depression via self-report measures: a review. Clin Interv Aging 13:2021-2044

36. Miller ME et al (2018) Gait speed and mobility disability: revisiting meaningful levels in diverse clinical populations. J Am Geriatr Soc 66:954-961

37. Corfield EC, Martin NG, Nyholt DR (2016) Co-occurrence and symptomatology of fatigue and depression. Compr Psychiatry 71:1-10

38. American Psychiatric Association (2013) Diagnostic and statistical manual of mental disorders, 5th edn. American Psychiatric Publishing,Arlington, VA

39. Dalle Carbonare L et al (2009) Physical disability and depressive symptomatology in an elderly population: a complex relationship. The Italian longitudinal study on aging (ILSA). Am J Geriatr Psychiatry 17:144-154

40. Groeneweg-Koolhoven I et al (2017) Apathy in early and late-life depression. J Affect Disord 223:76-81

41. Veronese $\mathbf{N}$ et al (2017) Frailty and incident depression in community-dwelling older people: results from the ELSA study. Int J Geriatr Psychiatry 32:e141-e149

42. Soysal P et al (2017) Relationship between depression and frailty in older adults: a systematic review and meta-analysis. Ageing Res Rev 36:78-87

43. Aprahamian I et al (2019) Depression is associated with self-rated frailty in older adults from an outpatient clinic: a prospective study. Int Psychogeriatrics 31:425-434

44. Tian Q et al (2020) Perception of energy and objective measures of physical activity in older adults. J Am Geriatr Soc. https://doi. org/10.1111/jgs.16577

45. Saunders JB, Inman VT, Eberhart HD (1953) The major determinants in normal and pathological gait. J Bone Jt Surg Am 35:543-558 
46. Simonsick EM, Montgomery PS, Newman AB et al (2001) Measuring fitness in healthy older adults: the health $\mathrm{ABC}$ long distance corridor walk. J Am Geriatr Soc 49:1544-1548

47. Loy BD, O'Connor PJ, Dishman RK (2013) The effect of a single bout of exercise on energy and fatigue states: a systematic review and meta-analysis. Fatigue Biomed Heal Behav 1:223-242

48. Herring MP, Fleming KM, Hayes SP et al (2017) Moderators of exercise effects on depressive symptoms in multiple sclerosis: a meta-regression. Am J Prev Med 53:508-518

49. Hong S, Dimsdale JE (2003) Physical activity and perception of energy and fatigue in obstructive sleep apnea. Med Sci Sport Exerc 35:1088-1092

50. Anthony JC, Niaz U, Von Korff MR et al (1982) Limits of the 'MiniMental State' as a screening test for dementia and delirium among hospital patients. Psychol Med 12:397-408
51. Siqueira GSA, Hagemann P, De MS et al (2019) Can MoCA and MMSE Be interchangeable cognitive screening tools? A systematic review. Gerontologist 59:e743-e763

Publisher's Note Springer Nature remains neutral with regard to jurisdictional claims in published maps and institutional affiliations. 